two species differ, as is the rule in the genus, much less than the males. The female variabilis has pale cilia on the tegulae and the fore tarsus blackened from the tip of the first joint, whereas the female germanus has black tegular cilia and only the terminal joint of the fore tarsus black. I have seen one female, the exception above alluded to, that had half of the tegular cilia yellow, and half of them black.

I am quite certain that the female germanus was known to Loew. He says, after mentioning the fact that $D$. variabilis is more variable in the coloring of the posterior tarsi than is usually the case with the species of the genus Dolichopus: "I possess also some other females which I can only distinguish from the above described $q$ of $D$. $v a^{-}$ riabilis by their black ciliated tegulae ${ }^{\text {. }}$ It seems therefore that either the females vary in the coloring of the cilia, or that we have here two exceedingly similar species." Loew with his usual care and circumspection did not venture to describe these females as a new species.

( $T$ o be continued.)

\title{
NOTES ON THE STRUCTURE AND HISTORY OF HAEMATOBIA SERRATA.
}

BY JOHN B. SMITH, NEW BRUNSWICK, N. J.

During the summer of 1889 the alarm occasioned by the appearance in excessive numbers of this species, known as the "Horn Fly," induced me to study some of the structures rather carefully to demonstrate the impossibility of injuries such as were charged to the fly. The result of these studies appeared in popular form in Bulletin 62 of the New Jersey Experiment Station, and as they cover some ground not heretofore trodden, I give some of them in a more technical and condensed form to the entomological public.

The mouth parts were more particularly studied - not so carefully as the studies made on some other species by Dr. Dimmock, but simply to show the gross anatomy. In this species the opercular sheath is a ringed structure, enlarged basally and roughly shaped like an "Indian Club." The tube is not complete, but there is an infolding above and in front of the sheath which, while making it practically tight, gives a wide range of motion in enlarging and and contracting. On the outer side this sheath is furnished with rather sparse hair set into deep pits, evidently tactile and specialized, and not mere pubescence. In general structure Stomoxys calcitrans which was studied 
comparatively, agrees with the present species so far as the sheath is concerned.
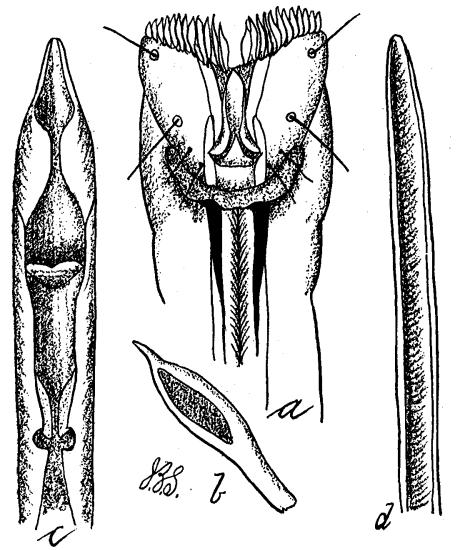

Fig. I.

Toward the tip the sheath becomes narrower and more closely infolded until just below the labellae a chitinous, curved, cross piece unites the two sides and makes the tube complete. Chitinous rods, quite heavy at their point of inception at this cross piece run upward close to the margin of the incurved edges, gradually losing themselves in the body of the sheath. S. calcitrans has a very similar structure. The extreme tip is somewhat tumid, smooth, set with a few tactile hairs and furnished toward the back with a series of fleshy processes which are apparently sucker discs, being hollow with an oval opening at the side near the tip. Centrally there is an elongate, somewhat trigonate opening, with corneous edges through which the hypopharynx (lancet) and perhaps the canula (lancet sheath) are extended when the insect pierces its prey; the soft lips with the fleshy suckers form a close union with the punctured surface.

$S$. calcitrans differs very decidedly in the structure of the tip, the lips not being tumid while the edge is completely encircled by the sucker discs.

The canula or axis piece or lancet sheath, carries the hypopharynx or true lancet. It is rather more than half a cylinder to near the tip, where there is a beautiful adaptation to hold and stiffen the lancet. The open sides of the canula

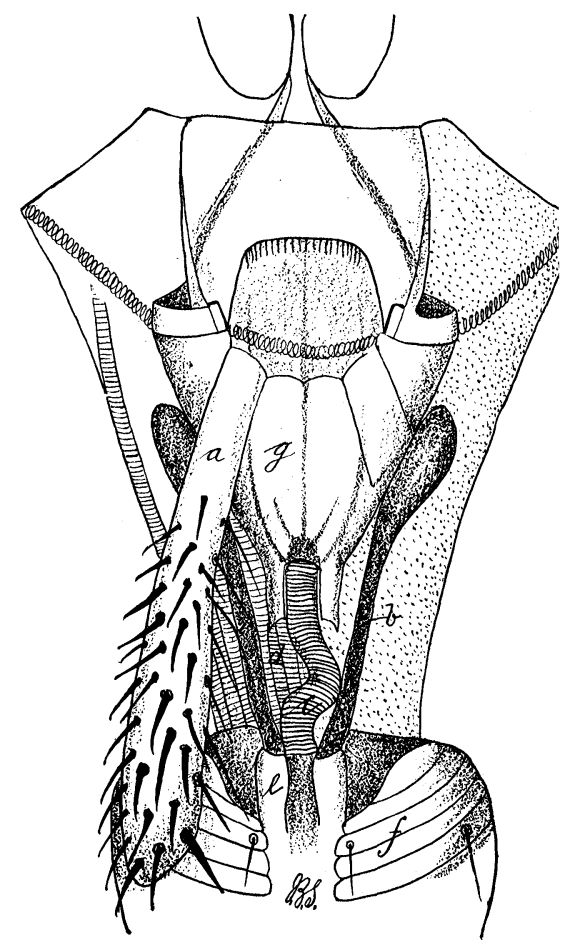

FIG. 2.

are for a short distance almost contiguous then again widely open, showing a transverse septum rising to half the 
height of the cylinder, with a central trigonate nick to guide the lancet, and finally again the tube is almost completed just before the tip. This tip has exactly the shape of the opening at the tip of the opercular sheath and fills it completely. It is perhaps questionable whether this organ is used in piercing or is even inserted into the wound. The hypopharynx or lancet is a simple half tube. In Stomoxys calcitrans the structure of the canula is different and much more simple, being uniform until just below the extreme tip and there the tube is completed.

The opercular sheath contains considerable muscular and other structure which was not studied. From the base of the hypopharynx a ringed tube leads direct into the fulcrum which is the sucking or pumping organ. From the base of the canula on each side the "great tendons" of Macloskie extend along the side of the fulcrum nearly to the top. These are according to Dimmock "the remnants of the basal chitinous supports of the maxilla." They are securely attached to the canula and I could not find any point of attachment at the other extremities. In separating the parts of the mouth they always remain attached to the canula. In specimens mounted in balsam no muscular structure is observable attached to them. The fulcrum is funnel shaped as shown in the figure, and to the upper margin the very long heavy palpi are attached. In Stomoxys calcitrans the fulcrum is quite different in shape, quadrate conic rather than cylindric, the very small palpi attached to the frontal lateral supporting rods rather close to the lower part of the fulcrum and therefore essentially different in location and more like Musca.

The palpi in Haematobia are quite densely set with stout spinous hair.

Dr. Macloskie is studying Stomoxys and Haematobia, and he will undoubtedly be able to complete and elaborate this rough outline structure.

In examining the wings I noticed a

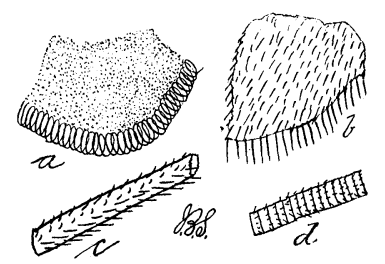

FIG. 3.

curious difference between the main wing and the alar appendix. The former is of the common membranous structure clothed with very fine pubescence, the margins ciliated and not thickened. The appendix shows no pubescence but is minutely and rather densely punctulate. The margin is formed by a spiral, coil-like tracheate structure. The veins also show differences in structure. The main trunks are complete, continuous tubes set with fine, rather short hair. The auxilliary veins which do not reach the base, and some of the transverse veins, are distinctly ringed and segmented, the segments set with transverse rows of minute hair. Whether this indicates the original tracheate nature of these veins I cannot say. 


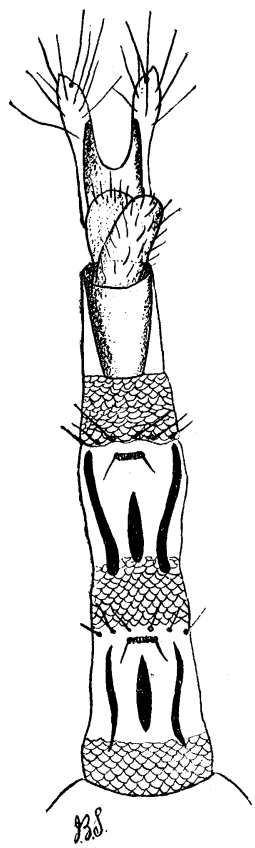

The extensile oviduct of the fly is a very pretty object when fully spread out under the microscope. It is seen that at each segment is a broad belt of closely laid scales and that strong chitinous rods support the tube and probably aid in the expulsion of the ovum.

I have examined this organ in a number of diptera and find it different in detail in each species.

The genitalia of the male are quite complicated, and in this species comparatively

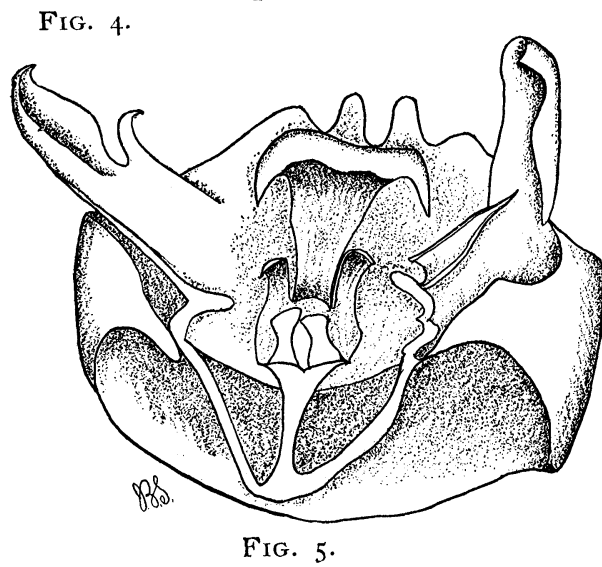

larger and more easily studied than in S. calcitrans or in Musca domestica, both of which differ very decidedly from our species. The figure is from a camera drawing and no attempt has been made to symmetrically arrange the parts. The broad uncus with its spreading and elongated lateral angles at tip is supported at hase by two smaller processes with rounded, curved tips. The harpes are membranous rather than chitinous, and are only apparently dissimilar, the hook like process of the left figure being concealed on the right. So the accessory clasper at the base of the right harpe is not shown on the left, though it is also present. A close study of these organs in the diptera will undoubtedly show many interesting structures.

The mouth parts of the larva were also studied and the figured structure was presented. The outward appearance is that of a ringed lip with a central opening behind which rise the fleshy eminences which bear the small palpi. The lips are made up of the usual tubular structure, the margins open and giving the appearance shown at 3 in figure 6 . It seems there as if the tubes were made of an endless piece of structure the material being drawn from one to the other. When properly treated the tissue becomes transparent and the chitinous sucking or pumping stomach is brought into view, lying mostly within the first segment. This organ is roughly six-sided, three of the plates distinctly chitinous, the others more membranous and furnished with powerful muscles-in fact muscular bands are attached at all angles of this structure and thus the pasty mixture upon which the larva subsists is drawn into the stomach. 
The fly oviposits, in my experience, largely at night, though the observations made under Prof. Riley's direction prove that many are also laid during the day. Flies in captivity always oviposited for me at night only. I never was able to see in the field a single fly on fresh droppings during the day, while eggs were easily found on these one day old. No

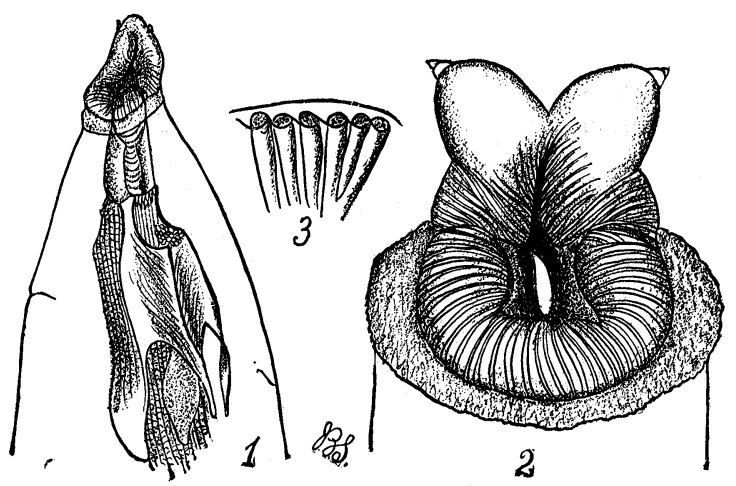

FIG. 6. amount of negative result can counterbalance positive observation, but I still believe that eggs are largely laid at night, in New Jersey at least. Observations made by Mr. Bodee of Freehold are confirmatory of this view. 'The fly hibernates in the imago state, and in a winter like the one just past, breeds nearly all the time, remaining quiet in stables and wherever it finds shelter only in really cold weather. Dr. Lockwood bred the flies in February from droppings brought in from the fields.

It is a rather curious phase in the history of this fly that up to the beginning of August it seems to increase enormously, fairly swarming about the cattle, - worst perhaps in June and early July, - while they are scarcely

annoying after the middle of August and hardly as abundant as Stomoxys. It seems also that early in the year they congregate about the base of the horns of cattle much more than they do later in the season.

Fig. 1. $a$, Tip of opercular sheath; $b$, a single sucker disc; $c$, tip of canula; $d$, tip of hypopharynx: all enlarged.

Fig. 2. a, Palpus - The corresponding organ of the opposite side is omitted; $b$, the "great tendons"; $c$, throat; $d$, muscular bands to direct the proboscis; $e$, base of canula; $f$, base of opercular sheath shoving ringed structure and tactile hairs; $g$, the fulcrum: enlarged.

Fig. 3. $a$, Alar appendix; $b$, piece of wing; $c$, piece of trunk vein; $d$, piece of transverse or auxilliary vein: enlarged.

Fig. 4. Oviduct of female: enlarged.

Fig. 5. Genitalia of male: enlarged.

Fig. $6 . I$, Head and anterior segment of larva showing structure of pumping stomach; 2, mouth, further enlarged; 3, tubes of lip still further enlarged.

\section{NOTES ON THE SOUTHERN DISTRI- BUTION OF SOME COMMON BUTTERFLIES}

There seems to be a little uncertainty as to the proper Southern range of some of our otherwise well-known butterflies, and so possibly the following notes, though coming from only a linited locality, may help to add a link in the chain of life history of our lepidoptera.

Chrysophanus hypophlatas: In the month of August I889, I found this fairly abundant in the neighborhood of Caesar's Ilead, S. C. These agree with New Jerse: 

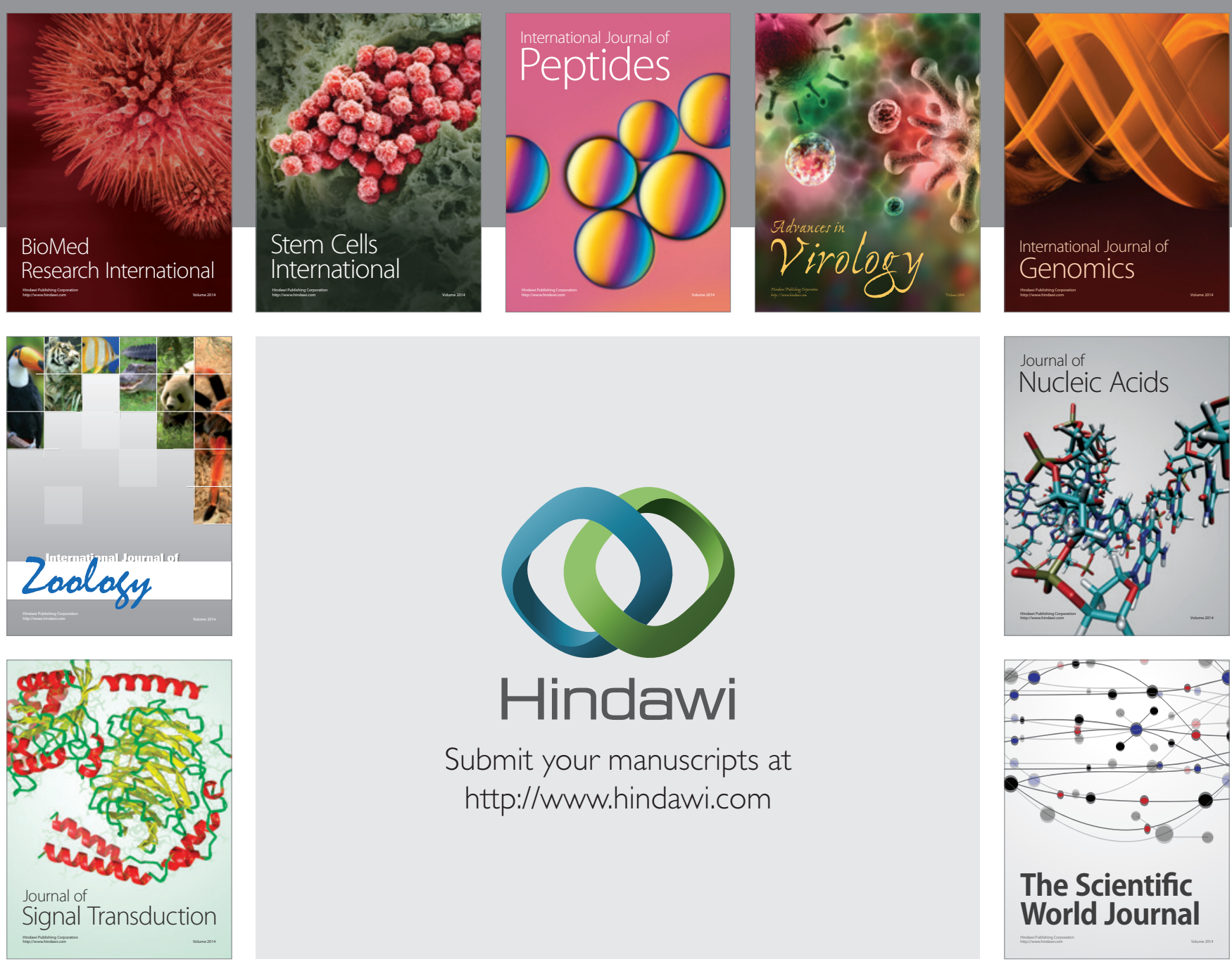

Submit your manuscripts at

http://www.hindawi.com
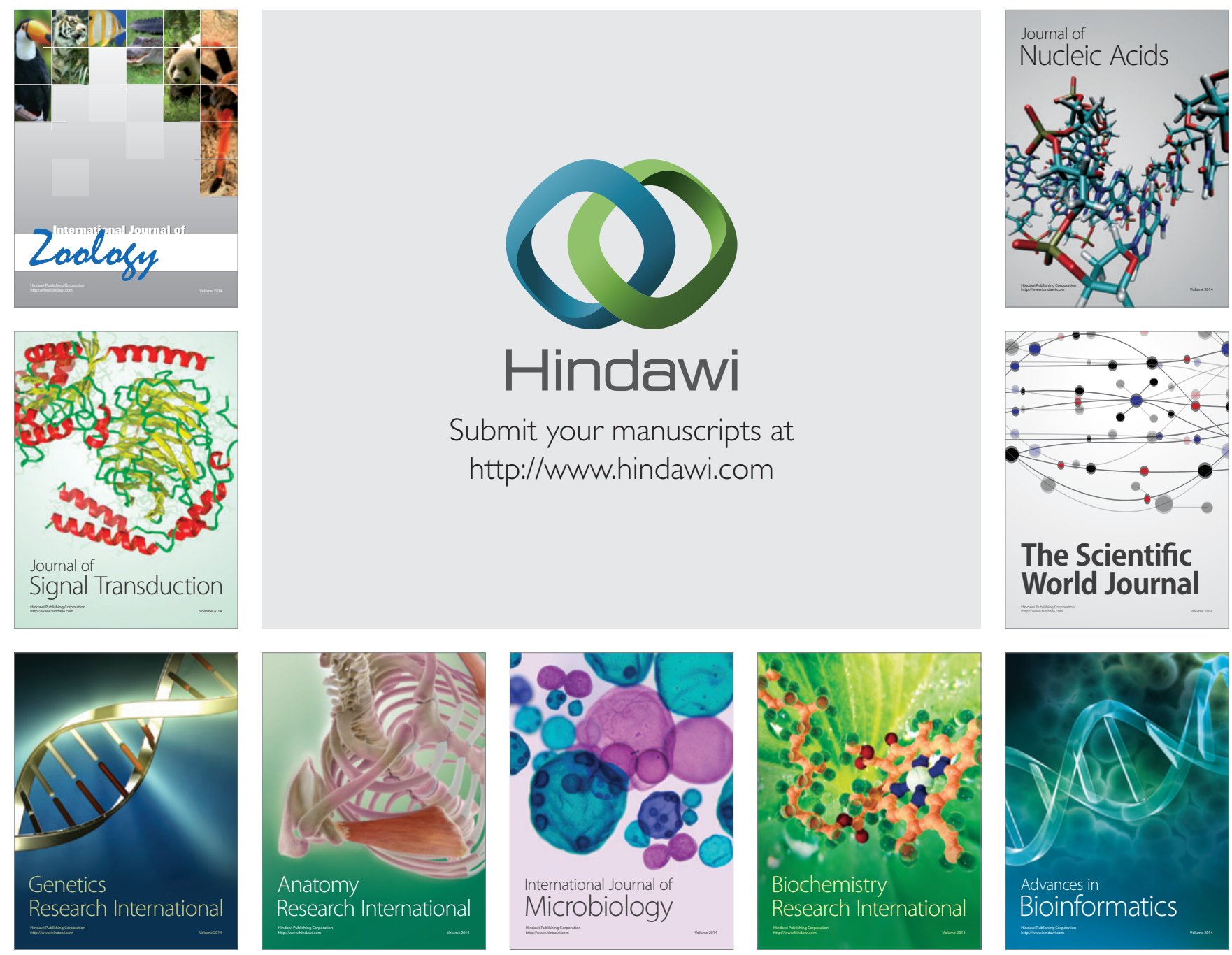

The Scientific World Journal
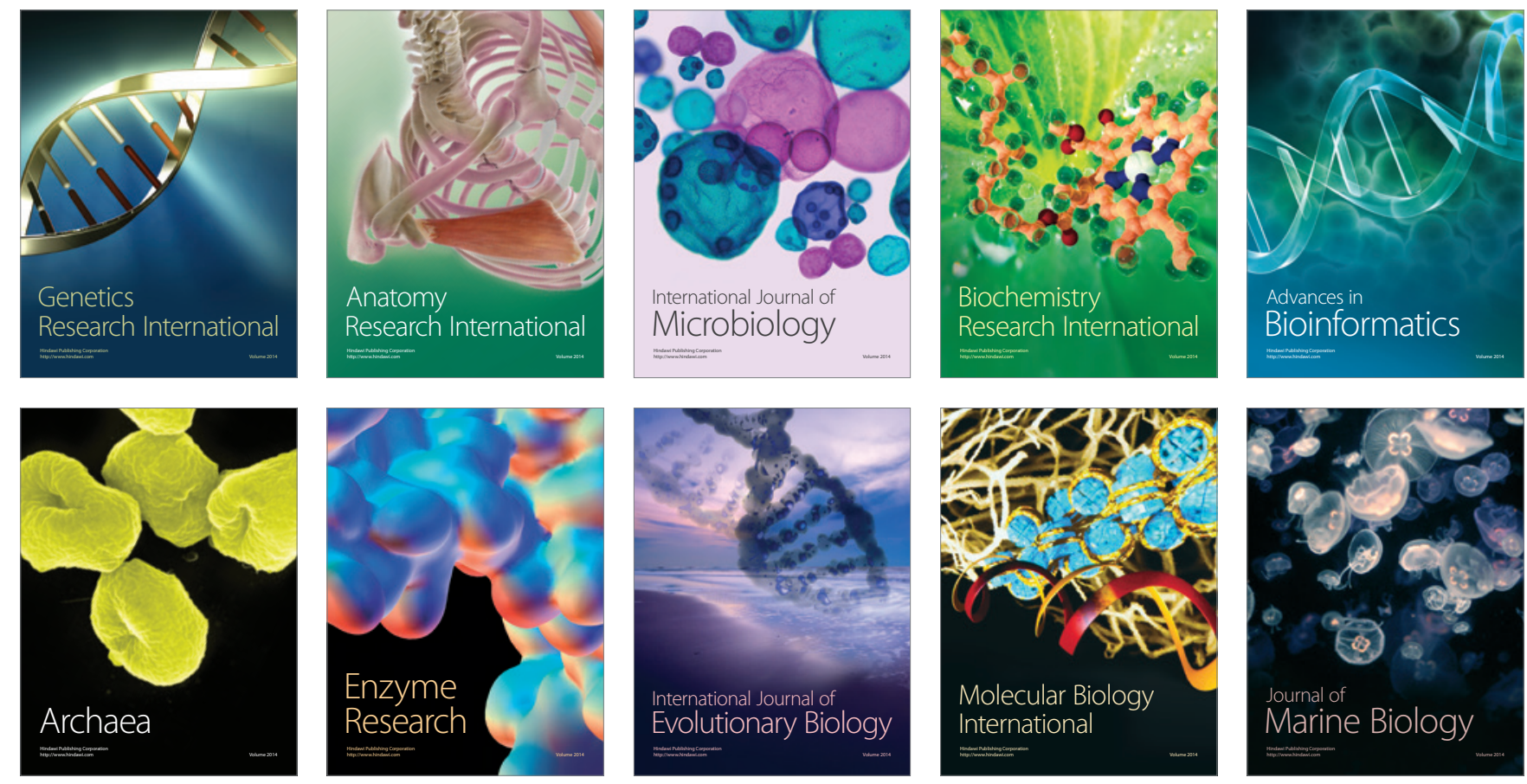